\title{
Serre Problem for Unbounded Pseudoconvex Reinhardt Domains in $\mathbb{C}^{2}$
}

\author{
Łukasz Kosiński
}

Received: 8 October 2009 / Published online: 10 August 2010

(C) Mathematica Josephina, Inc. 2010

\begin{abstract}
We give a characterization of non-hyperbolic pseudoconvex Reinhardt domains in $\mathbb{C}^{2}$ for which the answer to the Serre problem is positive. Moreover, all non-hyperbolic pseudoconvex Reinhardt domains in $\mathbb{C}^{2}$ with non-compact automorphism group are explicitly described.
\end{abstract}

Mathematics Subject Classification (2000) 32L05 · 32A07

Keywords Serre problem $\cdot$ Reinhardt domains

\section{Statement of Result}

Throughout this paper the class of Stein domains $D$ for which the answer to the Serre problem is positive (with the fiber equal to $D$ ) is denoted by $\mathfrak{S}$, i.e., $D \in \mathfrak{S}$ if for any Stein manifold $B$, any holomorphic fiber bundle $E \rightarrow B$ with the base $B$, and the Stein fiber $D$ is Stein. In 1953 J.-P. Serre raised the question of whether all Stein manifolds are in $\mathfrak{S}$.

Despite many positive results the answer to the Serre problem is in general negative. Actually, Skoda proved that $\mathbb{C}^{2} \notin \mathfrak{S}$ (see [20]). The counterexamples with bounded domains as fibers were found by G. Coeuré and J.-J. Loeb ([1]). In [17] P. Pflug and W. Zwonek gave a characterization of all hyperbolic Reinhardt domains of $\mathbb{C}^{2}$ not in $\mathfrak{S}$. Next, K. Oeljeklaus and D. Zaffran solved in [16] the Serre problem for bounded Reinhardt domains in $\mathbb{C}^{3}$. Recently, a classification result for bounded

Communicated by Alexander Isaev.

Research partially supported by the KBN grant $N^{\circ}$ N N201 271435 and by the foundation of A. Krzyżanowski.

Ł. Kosiński $(\bowtie)$

Instytut Matematyki, Uniwersytet Jagielloński, Łojasiewicza 6, 30-348 Kraków, Poland

e-mail: lukasz.kosinski@gazeta.pl 
Reinhardt domains of $\mathbb{C}_{*}^{d}$ for arbitrary $d \geq 2$ has been obtained by D. Zaffran in [23]. In particular, the case $\mathbb{C}_{*}^{d}, d \geq 2$, may be deduced from this result (it follows easily from [23], Main Theorem).

In this paper we deal with non-hyperbolic Reinhardt domains in $\mathbb{C}^{2}$ and we solve the Serre problem for them. The main goal is to show the following:

Theorem 1 Let $D$ be a pseudoconvex non-hyperbolic Reinhardt domain. Then $D \notin \mathfrak{S}$ if and only if $\mathbb{C}_{*}^{2} \subset D$ or $D$ is algebraically equivalent to a domain of the form

$$
\left\{\left(z_{1}, z_{2}\right) \in \mathbb{C}_{*}^{2}:\left|z_{1}\right|\left|z_{2}\right|^{p \pm \sqrt{q}}<1\right\}
$$

where $p, q \in \mathbb{Q}, q>0, \sqrt{q} \in \mathbb{R} \backslash \mathbb{Q}$.

Remark 2 Note that for the pseudoconvex Reinhardt domain $D$ the condition $\mathbb{C}_{*}^{2} \subset D$ means that $D \in\left\{\mathbb{C}^{2}, \mathbb{C}_{*}^{2}, \mathbb{C} \times \mathbb{C}_{*}, \mathbb{C}_{*} \times \mathbb{C}\right\}$.

The following result gives a description of non-hyperbolic pseudoconvex Reinhardt domains in $\mathbb{C}^{2}$ whose group of automorphisms is non-compact.

Theorem 3 Let $D$ be a non-hyperbolic pseudoconvex Reinhardt domain in $\mathbb{C}^{2}$. Then the group $\operatorname{Aut}(D)$ is non-compact if and only if the logarithmic image of the domain $D$ contains an affine line or (up to a permutation on components) $D$ is contained in $\mathbb{C} \times \mathbb{C}_{*},\{0\} \times \mathbb{C}_{*} \subset D$ and $\log D=\{(t, s): t<\psi(s), s \in \mathbb{R}\}$, where $\psi: \mathbb{R} \rightarrow \mathbb{R}$ is $a$ concave function satisfying the property $\psi(\beta+s)-\psi(s)=\alpha+k s, s \in \mathbb{R}$, for some $\alpha, \beta \in \mathbb{R}, \beta \neq 0, k \in \mathbb{Z}_{*}$.

Remark 4 An example of a function $\psi$ appearing in Theorem 3 is the function $\psi(s)=a s^{2}+b s+c, s \in \mathbb{R}$, where $a<0, b, c \in \mathbb{R}$.

\section{Preliminaries}

Here is some notation. By $\mathbb{D}$ we denote the open unit disc in the complex plane. Let $\mathbb{A}(r, R)=\{z \in \mathbb{C}: r<|z|<R\},-\infty<r<R<\infty, R>0$. Note that if $r>0$, then $\mathbb{A}(r, R)$ is an annulus. For simplicity we put $\mathbb{A}(r)=\mathbb{A}(1 / r, r), r>0$. Moreover, for a domain $D$ in $\mathbb{C}^{n}$, the set $D \backslash\{0\}$ is denoted by $D_{*}$.

For a Reinhardt domain $D \subset \mathbb{C}^{n}$ we define

$$
\log D=\left\{\left(x_{1}, \ldots, x_{n}\right) \in \mathbb{R}^{n}:\left(e^{x_{1}}, \ldots, e^{x_{n}}\right) \in D\right\} .
$$

It is well-known that, for any pseudoconvex domain in $\mathbb{C}^{n}$, its logarithmic image is convex.

Put

$$
\mathbb{C}(\lambda):= \begin{cases}\mathbb{C}, & \text { if } \lambda \geq 0, \\ \mathbb{C}_{*}, & \text { if } \lambda<0,\end{cases}
$$


and

$$
\mathbb{C}(\alpha):=\mathbb{C}\left(\alpha_{1}\right) \times \cdots \times \mathbb{C}\left(\alpha_{n}\right), \quad \alpha \in\left(\mathbb{R}^{n}\right) .
$$

Given $\alpha \in \mathbb{R}^{n}$ and $z \in \mathbb{C}(\alpha)$, we put $z^{\alpha}=z_{1}^{\alpha_{1}} \cdots z_{n}^{\alpha_{n}}$. ${ }^{1}$ Moreover, we define

$$
\varphi_{A}(z):=z^{A}:=\left(z^{A^{1}}, \ldots, z^{A^{m}}\right), \quad z \in \mathbb{C}(A):=\bigcap_{j=1}^{m} \mathbb{C}\left(A^{j}\right),
$$

where $A=\left(A_{k}^{j}\right)_{j=1, \ldots, m, k=1, \ldots, n} \in \mathbb{Z}^{m \times n}$.

Non-hyperbolic Reinhardt domains appearing in Theorem 1 have been characterized in the following way:

Theorem 5 (See [24]) Let D be a pseudoconvex Reinhardt domain in $\mathbb{C}^{n}$. Then the following conditions are equivalent:

- D is (Kobayashi, Carathéodory, or Brody) hyperbolic.

- D is algebraically equivalent to a bounded domain; in other words, there is an $A \in \mathbb{Z}^{n \times n}$ with $|\operatorname{det} A|=1$ such that $\varphi_{A}(D)$ is bounded and $\left.\left(\varphi_{A}\right)\right|_{D}$ is a biholomorphism onto the image.

- $\log D$ contains no straight lines, and $D \cap\left(\mathbb{C}^{j-1} \times\{0\} \times \mathbb{C}^{n-j}\right)$ is either empty or hyperbolic (viewed as domains in $\mathbb{C}^{n-1}$ ), $j=1, \ldots, n$.

In view of Theorem 5, non-hyperbolic pseudoconvex Reinhardt domains in $\mathbb{C}^{2}$ not containing $\mathbb{C}_{*}^{2}$ may be divided into two classes.

The first one contains non-hyperbolic, pseudoconvex Reinhardt domains $D$ such that $D \cap \mathbb{C}_{*}^{2}$ is hyperbolic.

The second one consists of domains whose logarithmic images contain a straight line. One may prove that such domains are algebraically equivalent to

$$
D_{\alpha, r_{-}, r_{+}}:=\left\{\left(z_{1}, z_{2}\right) \in \mathbb{C}(\alpha): r_{-}<\left|z_{1}\right|^{\alpha_{1}}\left|z_{2}\right|^{\alpha_{2}}<r_{+}\right\},{ }^{2}
$$

where $\alpha=\left(\alpha_{1}, \alpha_{2}\right) \in \mathbb{R}_{*}^{2},-\infty<r_{-}<r_{+}<\infty, r_{+}>0$.

Definition 6 A domain $D_{\alpha, r_{-}, r_{+}}$is said to be of rational type if $t \alpha \in\left(\mathbb{Q}^{2}\right)_{*}$ for some $t>0$

Otherwise, $D_{\alpha, r_{-}, r_{+}} \subset \mathbb{C}^{2}$ is said to be of irrational type.

It is clear that $D_{\alpha, r^{-}, r^{+}}$is algebraically equivalent to a domain of one of the following types:

\footnotetext{
${ }^{1}$ We define $0^{0}:=1$.

${ }^{2}$ Note that replacing $\left(\alpha_{1}, \alpha_{2}\right)$ by $\left(-\alpha_{1}, \alpha_{2}\right)$ allows us to add or to remove the axis $\{0\} \times \mathbb{C}$ in the case when $\alpha_{1} \neq 0$. In other words, $\left\{\left(z_{1}, z_{2}\right) \in \mathbb{C}_{*} \times \mathbb{C}\left(\alpha_{2}\right): r_{-}<\left|z_{1}\right|^{\alpha_{1}}\left|z_{2}\right|^{\alpha_{2}}<r_{+}\right\}$, where $\alpha_{1}>0, \alpha_{2} \in \mathbb{R}$ is also covered by this symbol (it is algebraically equivalent to $\left.D_{\left(-\alpha_{1}, \alpha_{2}\right), r_{-}, r_{+}}\right)$. If $\alpha_{1}=0$, then $D_{\alpha, r_{-}, r_{+}=}$ $\mathbb{A}\left(r_{-}, r_{+}\right) \times \mathbb{C}$. Moreover, $\mathbb{A}\left(r_{-}, r_{+}\right) \times \mathbb{C}_{*}$ is algebraically equivalent to $D_{(1,-1), r_{-}, r_{+}}$.
} 
(a) $D_{\beta}:=\left\{\left(z_{1}, z_{2}\right) \in \mathbb{C} \times \mathbb{C}(\beta):\left|z_{1}\right|\left|z_{2}\right|^{\beta}<1\right\}, \beta \in \mathbb{R}$.

(b) $D_{\beta}^{*}:=\left\{\left(z_{1}, z_{2}\right) \in \mathbb{C}_{*} \times \mathbb{C}(\beta): 0<\left|z_{1}\right|\left|z_{2}\right|^{\beta}<1\right\}, \beta \in \mathbb{R}_{\geq 0}$.

(c) $D_{\beta, r}:=\left\{\left(z_{1}, z_{2}\right) \in \mathbb{C} \times \mathbb{C}(\beta): 1 / r<\left|z_{1}\right|\left|z_{2}\right|^{\beta}<r\right\}, \beta \in \mathbb{R}_{\geq 0}, r>1$.

Remark 7 Observe that any Reinhardt domain $D_{\alpha, r_{-}, r_{+}}$of rational type is algebraically equivalent to one of the following domains:

(d) $\mathcal{D} \times \mathcal{C}$, where $\mathcal{D}$ is either a disc, a pointed disc, or an annulus, and $\mathcal{C} \in\left\{\mathbb{C}, \mathbb{C}_{*}\right\}$.

(e) $\left\{(z, w) \in \mathbb{C}^{2}:\left|z^{p}\right|\left|w^{q}\right|<1\right\}$, where $p, q \in \mathbb{N}$ are relatively prime.

(f) $\left\{(z, w) \in \mathbb{C}_{*} \times \mathbb{C}:\left|z^{p}\right|\left|w^{q}\right|<1\right\}$, where $p, q$ are relatively prime natural numbers, $(p, q) \neq(1,1)$.

Proof We will show that any domain of the form (a), (b), (c) with rational $\beta$ is algebraically equivalent to one of the domains enumerated above.

Domains of the form $D_{0}, D_{0}^{*}$, and $D_{0, r}$ are in (d).

Note that domains of the form (e) may be written as $D_{\frac{q}{p}}$. Moreover, domains of the form (f) are algebraically equivalent to $D_{-\frac{q}{p}}$. Therefore, any domain of the form (a) with $\beta \neq 0$ is of the form (e) or (f).

Fix a rational $\beta>0$ and assume that $\beta=p / q$, where $p$ and $q$ are relatively prime natural numbers. There are $m, n \in \mathbb{Z}$ such that $p m+q n=1$. Observe that the mapping $D_{\beta}^{*} \ni\left(z_{1}, z_{2}\right) \rightarrow\left(z_{1}^{q} z_{2}^{p}, z_{1}^{-m} z_{2}^{n}\right) \in \mathbb{D}_{*} \times \mathbb{C}_{*}$ is a biholomorphism.

Similarly, one may show that $D_{\beta, r}$ is algebraically equivalent to $\mathbb{A}(r) \times \mathbb{C}_{*}$.

What remains to do is to observe that the set $\left\{(z, w) \in \mathbb{C}_{*} \times \mathbb{C}:|z||w|<1\right\}$ is algebraically equivalent to $\mathbb{D} \times \mathbb{C}_{*}$.

Our paper is organized as follows. In Sect. 3 we give the proof of Theorem 1 for Reinhardt domains of the form (2) of the rational type.

Section 4 is devoted to solving the Serre problem for Reinhardt domains of the form (2) of irrational type. We obtain there a natural correspondence between the automorphisms of Reinhardt domains of irrational type and the famous Pell's equation.

In Sect. 5 we prove Theorem 1 for non-hyperbolic Reinhardt domains which are hyperbolic after removing the axes $(\mathbb{C} \times\{0\}) \cup(\{0\} \times \mathbb{C})$.

It seems probable to us that the solution of the Serre problem for $\mathbb{C} \times \mathbb{C}_{*}$ is known, however, we could not find it in the literature. Therefore, in Sect. 6 we present ideas explaining how to easily extend the procedure used in [3] to this domain.

Finally, in Sect. 7, using the obtained results we present the proofs of Theorems 1 and 3.

The two following theorems providing us with some classes of domains for which the answer to the Serre problem is positive will be useful. The first of them is the so-called Stehlé criterion.

Theorem 8 (See $[14,22])$ Let $D$ be a domain in $\mathbb{C}^{n}$. If there exists a real-valued plurisubharmonic exhaustion function $u$ on $D$ such that $u \circ f-u$ is bounded from above for any $f \in \operatorname{Aut}(D)$, then $D \in \mathfrak{S}$.

Observe that the Stehlé criterion is satisfied among others by domains whose group of automorphisms is compact. 
Theorem 9 (See [13]) Any open Riemann surface belongs to $\mathfrak{S}$.

Let us recall basic facts and definitions related to holomorphic fiber bundles which will be useful in the sequel. For more information we refer the reader to [6].

Let $E$ be an arbitrary holomorphic fiber bundle with the fiber $X$. The automorphisms of the form $\tau_{\alpha, \beta}=\tau_{\alpha} \circ \tau_{\beta}^{-1} \in \operatorname{Aut}\left(\left(\Omega_{\alpha} \cap \Omega_{\beta}\right) \times X\right)$, where $\tau_{\alpha}, \tau_{\beta}$ are trivializations (with associated domains $\Omega_{\alpha} \times X, \Omega_{\beta} \times X$, respectively) are so-called transition functions. It is clear that

$$
\tau_{\alpha, \beta} \circ \tau_{\beta, \gamma}=\tau_{\alpha, \gamma} \quad \text { on }\left(\Omega_{\alpha} \cap \Omega_{\beta} \cap \Omega_{\gamma}\right) \times X .
$$

On the other hand, having domains $\left(\Omega_{\alpha}\right)_{\alpha}$ and a family of functions $\left(\tau_{\alpha, \beta}\right)_{\alpha, \beta} \in$ $\operatorname{Aut}\left(\left(\Omega_{\alpha} \cap \Omega_{\beta}\right) \times X\right)$ satisfying the condition (3) we may define a holomorphic fiber bundle with the base $B=\bigcup_{\alpha} \Omega_{\alpha}$ and the fiber $X$ by putting

$$
E=\left(\bigsqcup_{\alpha}\left(\Omega_{\alpha} \times X\right)\right) / \sim,
$$

gluing the charts $\Omega_{\alpha} \times X$ via identification: $\left(x_{\alpha}, z_{\alpha}\right) \sim\left(x_{\beta}, z_{\beta}\right)$ if and only if $x_{\alpha}=$ $x_{\beta} \in \Omega_{\alpha} \cap \Omega_{\beta}$ and $\tau_{\alpha, \beta}\left(x_{\beta}, z_{\beta}\right)=\left(x_{\alpha}, z_{\alpha}\right)$.

Throughout this paper all holomorphic fiber bundles are understood to be of the form (4).

\section{Rational Case}

We start this section by collecting the formulas for automorphisms of Reinhardt domains of rational type. Some of them may be found in [9] and [21]. They may also be derived from the description of proper holomorphic mappings between Reinhardt domains of rational type obtained in [4] and [10]. As mentioned in Remark 7, any rational Reinhardt domain is algebraically equivalent to a domain of the form (d), (e), or (f).

\section{Theorem 10}

(1) The group of automorphisms of the domain $\left\{\left(z_{1}, z_{2}\right) \in \mathbb{C}^{2}:\left|z_{1} z_{2}\right|<1\right\}$ consists of the mappings

$$
\left(z_{1}, z_{2}\right) \rightarrow\left(z_{1} f\left(z_{1} z_{2}\right), e^{i \theta} z_{2} / f\left(z_{1} z_{2}\right)\right)
$$

and

$$
\left(z_{1}, z_{2}\right) \rightarrow\left(z_{2} f\left(z_{1} z_{2}\right), e^{i \theta} z_{1} / f\left(z_{1} z_{2}\right)\right)
$$

where $f \in \mathcal{O}^{*}(\mathbb{D})$ and $\theta \in \mathbb{R}$.

(2) The group of automorphisms of $\left\{\left(z_{1}, z_{2}\right) \in \mathbb{C}^{2}:\left|z_{1}^{p}\right|\left|z_{2}^{q}\right|<1\right\}$, where $p$ and $q$ are relatively prime natural numbers, $(p, q) \neq(1,1)$, consists of the mappings of the 
form

$$
\left(z_{1}, z_{2}\right) \rightarrow\left(a^{1 / p}\left(z_{1}^{p} z_{2}^{q}\right) z, e^{i \theta} \frac{z_{2}}{a^{1 / q}\left(z_{1}^{p} z_{2}^{q}\right)}\right)
$$

where $a \in \mathcal{O}^{*}(\mathbb{D}), \theta \in \mathbb{R}$.

(3) The group of automorphisms of the domain $\left\{\left(z_{1}, z_{2}\right) \in \mathbb{C}_{*} \times \mathbb{C}\right.$ : $\left.\left|z_{1}^{p}\right|\left|z_{2}^{q}\right|<1\right\}$, where $p$ and $q$ are relatively prime natural numbers, $p \geq 1, q \geq 2$, consists of the mappings of the form

$$
\left(z_{1}, z_{2}\right) \rightarrow\left(a^{1 / p}\left(z_{1}^{p} z_{2}^{q}\right) z_{1}, e^{i \theta} \frac{z_{2}}{a^{1 / q}\left(z_{1}^{p} z_{2}^{q}\right)}\right),
$$

where $a \in \mathcal{O}^{*}(\mathbb{D}), \theta \in \mathbb{R}$.

(4) Let $\mathcal{D} \subset \mathbb{C}$ be bounded and $\mathcal{C} \in\left\{\mathbb{C}, \mathbb{C}_{*}\right\}$. The group of automorphisms of $\mathcal{D} \times \mathcal{C}$ consists of the mappings

$$
\mathcal{D} \times \mathcal{C} \ni(\lambda, z) \rightarrow(a(\lambda), f(\lambda, z))
$$

where $a \in \operatorname{Aut}(\mathcal{D})$.

In the case when $\mathcal{C}=\mathbb{C}$ the mapping $f$ is of the form

$$
f(\lambda, z)=b(\lambda) z+c(\lambda), \quad \lambda \in \mathcal{D}, z \in \mathbb{C},
$$

where $b \in \mathcal{O}^{*}(\mathcal{D})$ and $c \in \mathcal{O}(\mathcal{D})$.

If $\mathcal{C}=\mathbb{C}_{*}$, then the mapping $f$ is of the form

$$
f(\lambda, z)=b(\lambda) z^{\epsilon}, \quad \lambda \in \mathcal{D}, z \in \mathbb{C}_{*},
$$

where $b \in \mathcal{O}^{*}(\mathcal{D})$ and $\epsilon= \pm 1$.

The following lemma due to Stehlé will be needed.

Lemma 11 ([22]) Let $B$ be a Stein manifold. Then for any covering $\left\{U_{\alpha}\right\}$ of $B$ there is a locally finite covering $\left\{B_{j}\right\}_{j \in \mathbb{N}}$ and a family of strictly plurisubharmonic, continuous functions $h_{j}$ in a neighborhood of $\overline{C_{j}}$, where $C_{j}=\bigcup_{i \leq j} B_{i}$, such that

(i) each $B_{j}$ is relatively compact in some $U_{\alpha}$,

(ii) $C_{j}$ is Stein for any $j$,

(iii) $h_{j}<0$ in $C_{j} \backslash B_{j}$ and $h_{j}>1$ in the neighborhood (in $C_{j}$ ) of $\overline{C_{j} \backslash C_{j-1}} \cap C_{j}$, $j \in \mathbb{N}$.

Theorem 12 Any Reinhardt domain $D$ in $\mathbb{C}^{2}$ of the form (2) of rational type belongs to the class $\mathfrak{S}$.

Remark 13 Note that the function satisfying the Stehle criterion does not exist for any domain appearing in the previous theorem. As an example, let us take $\mathbb{D} \times \mathbb{C}$. It is sufficient to observe that for any $f \in \mathcal{O}\left(\mathbb{D}, \mathbb{C}_{*}\right)$ the mapping

$$
\mathbb{D} \times \mathbb{C} \ni(\lambda, z) \rightarrow(\lambda, f(\lambda) z) \in \mathbb{D} \times \mathbb{C}
$$


is an automorphism of $\mathbb{D} \times \mathbb{C}$, and the growth of the function $f$ may be arbitrarily fast.

Proof of Theorem 12 The proof is divided into four cases.

(a) First we focus our attention on the case when the fiber is equal to $D=\{(z, w) \in$ $\left.\mathbb{C}^{2}:|z||w|<1\right\}$.

We will modify the idea used by Stehlé in the proof of Theorem 8 . Let $\pi: E \rightarrow B$ be a holomorphic fiber bundle with a Stein base $B$ and a fiber $D$. As we have already mentioned, $E$ is assumed to be given by the formula (4).

Using the description of the group of automorphisms of the domain $D$ we get that for any $b \in \Omega_{\alpha} \cap \Omega_{\beta}$ a transition function $\tau_{\alpha, \beta}$ is of one of two following forms:

$$
\left\{\begin{array}{l}
\tau_{\alpha, \beta}(b, z, w)=\left(b, z f_{\alpha, \beta}(b, z w), e^{i \theta_{\alpha, \beta}(b)} w / f_{\alpha, \beta}(b, z w)\right) \quad \text { or } \\
\tau_{\alpha, \beta}(b, z, w)=\left(b, w f_{\alpha, \beta}(b, z w), e^{i \theta_{\alpha, \beta}(b)} z / f_{\alpha, \beta}(b, z w)\right)
\end{array}\right.
$$

$((z, w) \in D)$, for some $f_{\alpha, \beta}(b, \cdot) \in \mathcal{O}^{*}(\mathbb{D})$ and $\theta_{\alpha, \beta}(b) \in \mathbb{R}$. Since $\tau_{\alpha, \beta}$ is holomorphic it follows that the mapping $\Omega_{\alpha} \cap \Omega_{\beta} \ni b \rightarrow e^{i \theta_{\alpha, \beta}(b)} \in \partial \mathbb{D}$ is holomorphic and therefore locally constant.

Let $\tilde{\pi}: \tilde{E} \rightarrow B$ denote a holomorphic fiber bundle with the base $B$ and the fiber $\mathbb{D}$ whose transition functions are defined by $\tilde{\tau}_{\alpha, \beta}(b, \lambda)=\left(b, e^{i \theta_{\alpha, \beta}(b)} \lambda\right), b \in \Omega_{\alpha} \cap \Omega_{\beta}$, $\lambda \in \mathbb{D}$. It follows from Theorem 9 that $\tilde{E}$ is Stein. Applying Lemma 8 to the family $\left\{\tilde{\pi}^{-1}\left(\Omega_{\alpha}\right)\right\}$ we get a locally finite covering $\left\{B_{j}\right\}$ and a family of strictly plurisubharmonic continuous functions $\left\{h_{j}\right\}$ satisfying conditions (i)-(iii) of Lemma 11. Let

$$
p: E \ni[(b, z, w)] \rightarrow[(b, z w)] \in \tilde{E}
$$

be a natural surjection between $E$ and $\tilde{E}$.

For $j \in \mathbb{N}$ choose $\alpha_{j}$ such that $B_{j}$ is relatively compact in $\tilde{\pi}^{-1}\left(\Omega_{\alpha_{j}}\right)$. Let $\tau_{j}:=\tau_{\alpha_{j}}$ denote any trivialization of the fiber bundle $\pi: E \rightarrow B$ defined on $p^{-1}\left(\tilde{\pi}^{-1}\left(\Omega_{\alpha_{j}}\right)\right)$. Put

$$
l(b, z, w)=\max \left\{\log ^{+}|z|, \log ^{+}|w|\right\}, \quad(b, z, w) \in B \times \mathbb{C}^{2} .
$$

The choice of $\left\{B_{j}\right\}$ and a standard compactness argument guarantee that for any $i, j \in \mathbb{N}$

$$
\sup \left\{l\left(\tau_{i} \circ \tau_{j}^{-1}(b, z, w)\right)-l(b, z, w):[b, z, w] \in p^{-1}\left(B_{i} \cap B_{j}\right)\right\}<\infty .
$$

Put

$$
v_{1}(x)=\exp \left(h_{1}(p(x))+l\left(\tau_{1}(x)\right)\right), \quad x \in p^{-1}\left(B_{1}\right) .
$$

Condition (5) allows us to choose constants $d \in(0,1)$ and $M>0$ such that

$$
d M \exp \left(l\left(\tau_{2}(x)\right)\right) \leq v_{1}(x) \leq 2 M \exp \left(l\left(\tau_{2}(x)\right)\right), \quad x \in p^{-1}\left(B_{1} \cap B_{2}\right) .
$$

Define

$$
\tilde{v}_{2}(x)=2 M \exp \left(l\left(\tau_{2}(x)\right)\right) \exp \left(\left(1-h_{2}(p(x))\right) \log \frac{d}{2}\right), \quad x \in p^{-1}\left(B_{2}\right) .
$$


It follows from the choice of $d, M$ that if $h_{2}(p(x))<0, x \in p^{-1}\left(B_{1} \cap B_{2}\right)$, then $\tilde{v}_{2}(x)<v_{1}(x)$. Moreover, if $h_{2}(p(x))>1, x \in p^{-1}\left(B_{1} \cap B_{2}\right)$, then $\tilde{v}_{2}(x)>v_{1}(x)$. Therefore, putting

$$
v_{2}(x)= \begin{cases}v_{1}(x), & x \in p^{-1}\left(B_{1} \backslash B_{2}\right), \\ \max \left\{v_{1}(x), \tilde{v}_{2}(x)\right\}, & x \in p^{-1}\left(B_{1} \cap B_{2}\right), \\ \tilde{v}_{2}(x), & x \in p^{-1}\left(B_{2} \backslash B_{1}\right),\end{cases}
$$

we obtain a plurisubharmonic continuous function defined on $p^{-1}\left(B_{1} \cup B_{2}\right)=$ $p^{-1}\left(C_{2}\right)$.

Similarly, let $d^{\prime} \in(0,1), M^{\prime}>0$ be such that

$$
d M \exp \left(l\left(\tau_{3}(x)\right)\right) \leq v_{2}(x) \leq 2 M \exp \left(l\left(\tau_{3}(x)\right)\right), \quad x \in p^{-1}\left(B_{3} \cap C_{2}\right) .
$$

Let us define

$$
\tilde{v}_{3}(x)=2 M^{\prime} \exp \left(l\left(\tau_{3}(x)\right)\right) \exp \left(1-h_{3}(p(x)) \log \frac{d^{\prime}}{2}\right) \quad \text { for } x \in p^{-1}\left(B_{3}\right),
$$

and

$$
v_{3}(x)= \begin{cases}v_{2}(x), & x \in p^{-1}\left(C_{2} \backslash B_{3}\right), \\ \max \left\{v_{2}(x), \tilde{v}_{3}(x)\right\}, & x \in p^{-1}\left(C_{2} \cap B_{3}\right), \\ \tilde{v}_{3}(x), & x \in p^{-1}\left(B_{3} \backslash C_{2}\right) .\end{cases}
$$

Exactly as before, we check that $v_{3} \in \mathcal{P} \mathcal{S H}\left(p^{-1}\left(C_{3}\right)\right)$.

Repeating this procedure inductively one may obtain a sequence of functions $v_{j} \in$ $\mathcal{P S H}\left(p^{-1}\left(C_{j}\right)\right)$ such that $v_{j} \leq v_{j+1}$ on $p^{-1}\left(C_{j}\right)$ and $v_{j}=v_{j+1}$ on $p^{-1}\left(C_{j} \backslash B_{j+1}\right)$.

Since the covering $\left\{B_{j}\right\}$ is locally finite, putting $v=\lim _{j} v_{j}$ we define properly a plurisubharmonic function on $E$.

Let $\tilde{u}$ be a strictly plurisubharmonic continuous exhaustion function on $\tilde{E}$. It follows from the construction of the function $v$ that

$$
u=\max \{\tilde{u} \circ p, v\}
$$

is a plurisubharmonic continuous exhaustion function on $E$.

Now, in order to show the Steinness of $E$ it suffices to repeat the argument used by N. Mok in the proof of the improvement of the Stehle criterion (see [14], Appendix III).

(b) Let us consider the case when the fiber $D$ is of the form $D=\{(z, w) \in$ $\left.\mathbb{C}^{2}:|z|^{p}|w|^{q}<1\right\}$ for some natural relatively prime $p, q,(p, q) \neq(1,1)$.

Let $\pi: E \rightarrow B$ be a holomorphic fiber bundle with the fiber $D$. Using Theorem 10 we infer that the transition functions are of the form

$$
\tau_{\alpha, \beta}(b, z, w)=\left(b, a_{\alpha, \beta}^{1 / p}\left(b, z^{p} w^{q}\right) z, e^{i \theta_{\alpha, \beta}(b)} \frac{w}{a_{\alpha, \beta}^{1 / q}\left(b, z^{p} w^{q}\right)}\right),
$$


where $a_{\alpha, \beta} \in \mathcal{O}^{*}\left(\left(\Omega_{\alpha} \cap \Omega_{\beta}\right) \times \mathbb{D}\right)$ and $\theta_{\alpha, \beta}(b) \in \mathbb{R}$. Moreover, it may be shown that $e^{i \theta_{\alpha, \beta}}$ is holomorphic and therefore locally constant.

Let $\tilde{\pi}: \tilde{E} \rightarrow B$ be a holomorphic fiber bundle with a fiber equal to $\tilde{D}=\{(z, w) \in$ $\left.\mathbb{C}^{2}:|z||w|<1\right\}$ and whose transition functions are defined in the following way:

$$
\tilde{\tau}_{\alpha, \beta}(b, z, w)=\left(b, a_{\alpha, \beta}(b, z w) z, e^{i q \theta_{\alpha, \beta}(b)} \frac{w}{a_{\alpha, \beta}(b, z w)}\right) .
$$

A direct computation allows us to observe that

$$
E \in[(b, z, w)] \rightarrow\left[\left(b, z^{p}, w^{q}\right)\right] \in \tilde{E}
$$

is a well defined proper holomorphic mapping. Therefore, by a result of Narasimhan in [15] (see also [5]) the manifold $E$ is Stein if and only if $\tilde{E}$ is Stein. However, the Steinness of $\tilde{E}$ follows from the previous case.

(c) Now we will show that $\mathcal{D} \times \mathcal{C} \in \mathfrak{S}$ for $\mathcal{D} \in\left\{\mathbb{D}, \mathbb{D}_{*}, \mathbb{A}(r)\right\}$.

Suppose that $E$ is a holomorphic fiber bundle with the fiber $\mathcal{D} \times \mathcal{C}$ and the Stein base $\Omega=\bigcup_{\alpha} \Omega_{\alpha}$. By Theorem 10, any transition function of $E$ is of the form

$$
\tau_{\alpha, \beta}(b, \lambda, z)=\left(b, m_{\alpha, \beta}(b, \lambda), f_{\alpha, \beta}(b, \lambda, z)\right), \quad(b, \lambda, z) \in\left(\Omega_{\alpha} \cap \Omega_{\beta}\right) \times \mathcal{D} \times \mathcal{C} .
$$

It follows from Theorem 10 that $m_{\alpha, \beta}(x, \cdot) \in \operatorname{Aut}(\mathcal{D})$ for any $x \in \Omega_{\alpha} \cap \Omega_{\beta}$ (it may be shown that $m_{\alpha, \beta}(x, \cdot)$ does not depend on $x$ on connected components of $\Omega_{\alpha} \cap \Omega_{\beta}$ ).

Let $\tilde{E}$ be a holomorphic fiber bundle with the base $\Omega$ and the fiber $\mathcal{D}$, whose transition functions $\tilde{\tau}_{\alpha, \beta} \in \operatorname{Aut}\left(\left(\Omega_{\alpha} \cap \Omega_{\beta}\right) \times \mathcal{D}\right)$ are given by the formulas

$$
\tilde{\tau}_{\alpha, \beta}(b, \lambda)=\left(b, m_{\alpha, \beta}(b, \lambda)\right), \quad(b, \lambda) \in\left(\Omega_{\alpha} \cap \Omega_{\beta}\right) \times \mathcal{D} .
$$

By Theorem 9, $\tilde{E}$ is Stein.

Now it is sufficient to observe that

$$
E \ni[(x,(\lambda, z))] \rightarrow[(x, \lambda)] \in \tilde{E}
$$

forms a holomorphic fiber bundle with the base $\tilde{E}$ and the fiber equal to $\mathcal{C}$. Using again Theorem 9 we get the Steinness of the bundle $E$.

(d) To finish the proof of the theorem, it suffices to show that $D=\left\{(z, w) \in \mathbb{C}_{*} \times\right.$ $\left.\mathbb{C}:\left|z^{p}\right|\left|w^{q}\right|<1\right\} \in \mathfrak{S}$, where $p, q \geq 1$ are relatively prime natural numbers, $(p, q) \neq$ $(1,1)$. We proceed similarly as in case (b). Namely, once again we aim at reducing the situation to the already solved case (c).

Observe that $D=\left\{(z, w) \in \mathbb{C}_{*} \times \mathbb{C}:\left|z^{p}\right||w|<1\right\}, p \geq 2$ is algebraically equivalent to $\mathbb{D} \times \mathbb{C}_{*}$ (take the mapping $\left.D \ni(z, w) \rightarrow\left(z^{p} w, w\right) \in \mathbb{D} \times \mathbb{C}_{*}\right)$. Therefore, we may assume that $q \geq 2$.

Suppose that $\pi: E \rightarrow B$ is a holomorphic fiber bundle. Then its transition functions must be of the form

$$
\begin{aligned}
& \tau_{\alpha, \beta}(b, z, w)=\left(b, z a_{\alpha, \beta}^{1 / p}\left(b, z^{p} w^{q}\right), e^{i \theta_{\alpha, \beta}(b)} w a_{\alpha, \beta}^{-1 / q}\left(b, z^{p} w^{q}\right)\right), \\
& (b, z, w) \in\left(\Omega_{\alpha} \cap \Omega_{\beta}\right) \times D,
\end{aligned}
$$


for some $a_{\alpha, \beta} \in \mathcal{O}^{*}\left(\left(\Omega_{\alpha} \cap \Omega_{\beta}\right) \times \mathbb{D}\right)$ and $\theta_{\alpha, \beta}(b) \in \mathbb{R}$. It is seen that $e^{i \theta_{\alpha, \beta}}$ may be chosen to be constant on connected components of $\Omega_{\alpha} \cap \Omega_{\beta}$. Therefore, putting

$$
\tilde{\tau}_{\alpha, \beta}(b, \lambda, z)=\left(b, e^{i q \theta_{\alpha, \beta}(b)} \lambda, z a_{\alpha, \beta}^{1 / p}(b, \lambda)\right), \quad(b, \lambda, z) \in\left(\Omega_{\alpha} \cap \Omega_{\beta}\right) \times \mathbb{D} \times \mathbb{C}_{*},
$$

we obtain a holomorphic fiber bundle $\tilde{E}$ with the base $\Omega$ and the fiber $\mathbb{D} \times \mathbb{C}_{*}$ such that a mapping given by the formula

$$
E \ni[(x, z, w)] \rightarrow\left[\left(x, z^{p} w^{q}, z\right)\right] \in \tilde{E}
$$

is proper and holomorphic.

The argument used in the proof of (b) finishes the proof of this case.

\section{Irrational Case}

The geometry of Reinhardt domains has been investigated in several papers (see $[18,19]$ and references contained there).

In [10] the author obtained a complete characterization of proper holomorphic mappings between Reinhardt domains of irrational type. This characterization is of key importance for our considerations. Therefore, in Theorem 14 we collect the most crucial part of it.

Using Theorem 14 we shall obtain in the proof of Theorem 15 a full description of the group of automorphisms of Reinhardt domains of irrational type. As mentioned before, it turns out that this problem is connected with the famous Pell's equation.

Recall that any Reinhardt domain of irrational type is equivalent to a domain of the type (a), (b), or (c).

Theorem 14 ([10], see also [19]) (i)

(a) If $\alpha \in \mathbb{R} \backslash \mathbb{Q}$, then the set of proper holomorphic mappings between $D_{\alpha, r}$ and $D_{\beta, R}$ is non-empty if and only if

$$
\frac{\log R}{\log r} \in \mathbb{Z}+\beta \mathbb{Z} \quad \text { and } \quad \alpha \frac{\log R}{\log r} \in \mathbb{Z}+\beta \mathbb{Z} .
$$

(b) Let $\alpha, \beta \in \mathbb{R} \backslash \mathbb{Q}$, and $r, R>1$ be such that $\frac{\log R}{\log r}=k_{1}+l_{1} \beta$ and $\alpha \frac{\log R}{\log r}=k_{2}+l_{2} \beta$ for some integers $k_{i}, l_{i}, i=1,2$. Then any proper holomorphic mapping $f$ : $D_{\alpha, r} \rightarrow D_{\beta, R}$ is one of the two following forms:

$$
\left\{\begin{array}{l}
f(z)=\left(a z_{1}^{k_{1}} z_{2}^{k_{2}}, b z_{1}^{l_{1}} z_{2}^{l_{2}}\right) \quad \text { or } \\
f(z)=\left(a z_{1}^{-k_{1}} z_{2}^{-k_{2}}, b z_{1}^{-l_{1}} z_{2}^{-l_{2}}\right)
\end{array} \quad z=\left(z_{1}, z_{2}\right) \in D_{\alpha, r},\right.
$$

where $a, b \in \mathbb{C}$ satisfy the equality $|a||b|^{\beta}=1$.

(ii) Let $\alpha, \beta \in \mathbb{R} \backslash \mathbb{Q}$. The set of proper holomorphic mappings between $D_{\alpha}^{*}$ and $D_{\beta}^{*}$ is non-empty if and only if $\alpha=\left(k_{2}+\beta l_{2}\right) /\left(k_{1}+\beta l_{1}\right)$ for some $k_{i}, l_{i} \in \mathbb{Z}, i=$ 
1,2. Moreover, if $\alpha=\left(k_{2}+\beta l_{2}\right) /\left(k_{1}+\beta l_{1}\right)$, where $k_{1}+l_{1} \beta>0$, then any proper holomorphic mapping $f: D_{\alpha}^{*} \rightarrow D_{\beta}^{*}$ is of the form

$$
f\left(z_{1}, z_{2}\right)=\left(a z_{1}^{k_{1}} z_{2}^{k_{2}}, b z_{1}^{l_{1}} z_{2}^{l_{2}}\right), \quad\left(z_{1}, z_{2}\right) \in D_{\alpha}^{*},
$$

where $a, b \in \mathbb{C}$ satisfy the equality $|a||b|^{\beta}=1$.

(iii) Let $\alpha, \beta \in \mathbb{R} \backslash \mathbb{Q}$.

(a) If $\alpha>0, \beta>0$, then the set $\operatorname{Prop}\left(D_{\alpha}, D_{\beta}\right)^{3}$ is non-empty if and only if $\alpha=p \beta$ for some $p \in \mathbb{Q}_{>0}$. In this case, all proper holomorphic mappings between $D_{\alpha}$ and $D_{\beta}$ are of the form

$$
\left(z_{1}, z_{2}\right) \rightarrow\left(z_{1}^{k}, z_{2}^{l}\right)
$$

where $k, l$ are positive integers such that $p=\frac{l}{k}$.

(b) If $\alpha<0, \beta<0$, then the set $\operatorname{Prop}\left(D_{\alpha}, D_{\beta}\right)$ is non-empty if and only if $\alpha=p_{1}+$ $p_{2} \beta$ for some rational $p_{1}, p_{2}, p_{2} \neq 0$. In this case, proper holomorphic mappings between $D_{\alpha}$ and $D_{\beta}$ are of the form

$$
\left(z_{1}, z_{2}\right) \rightarrow\left(z_{1}^{k_{1}} z_{2}^{k_{2}}, z_{2}^{l}\right)
$$

where $k_{1}, k_{2}, l, k_{1}>0$, are integers such that $p_{1}=\frac{k_{2}}{k_{1}}, i p_{2}=\frac{l}{k_{1}}$.

(c) If $\alpha \beta<0$, then there is no proper holomorphic mapping between $D_{\alpha}$ and $D_{\beta}$.

Our aim in this part of the paper is to show the following:

\section{Theorem 15 Let $\alpha \in \mathbb{R} \backslash \mathbb{Q}$. Then}

(a) $D_{\alpha}^{*} \notin \mathfrak{S}$ if and only if $\alpha=p \pm \sqrt{q}$ for some $p, q \in \mathbb{Q}, q>0$,

(b) $D_{\alpha} \in \mathfrak{S}$ for any $\alpha \in \mathbb{R} \backslash \mathbb{Q}$,

(c) $D_{\alpha, r} \in \mathfrak{S}$ for any $\alpha \in \mathbb{R} \backslash \mathbb{Q}, r>1$.

Proof (a) It follows from Theorem 14 that every automorphism of the domain $D_{\alpha}^{*}$ is of the form

$$
\left(z_{1}, z_{2}\right) \rightarrow\left(a z_{1}^{k_{1}} z_{2}^{k_{2}}, b z_{1}^{l_{1}} z_{2}^{l_{2}}\right),
$$

where $|a||b|^{\alpha}=1$ and $k_{1}, k_{2}, l_{1}, l_{2} \in \mathbb{Z}$ are such that $k_{1} l_{2}-k_{2} l_{1}= \pm 1, \alpha=\left(k_{2}+\right.$ $\left.l_{2} \alpha\right) /\left(k_{1}+l_{1} \alpha\right)$ and $k_{1}+l_{1} \alpha>0$.

These conditions imply that if $\alpha$ is not of the form $p \pm \sqrt{q}$ for any rational $p, q$, then $k_{1}=l_{2}=1$ and $k_{2}=l_{1}=0$. In other words, any automorphism of $D_{\alpha}^{*}$ is of the form $\left(z_{1}, z_{2}\right) \rightarrow\left(a z_{1}, b z_{2}\right), a, b \in \mathbb{C},|a||b|^{\alpha}=1$. Therefore, the plurisubharmonic function $u$ given by the formula

$$
u\left(z_{1}, z_{2}\right)=\max \left\{\frac{1}{1-\left|z_{1}\right|\left|z_{2}\right|^{\alpha}}, \log \left|z_{1}\right|,-\log \left|z_{1}\right|, \log \left|z_{2}\right|,-\log \left|z_{2}\right|\right\},
$$

$\left(z_{1}, z_{2}\right) \in D_{\alpha}^{*}$, satisfies the Stehlé criterion, hence $D_{\alpha}^{*} \in \mathfrak{S}$.

\footnotetext{
${ }^{3} \operatorname{Prop}(D, G)$ denotes the set of proper holomorphic mappings between $D$ and $G$.
} 
Suppose now that $\alpha=\frac{p}{n} \pm \sqrt{\frac{q}{n^{2}}}$ for some $p, q \in \mathbb{Z}, q>0, n \in \mathbb{N}$.

We are looking for $k_{i}, l_{i} \in \mathbb{Z}, k_{1}+\alpha l_{1} \geq 0$ such that (20) defines an automorphism of $D_{\alpha}^{*}$. Then the following equations are satisfied:

$$
\left\{\begin{array}{l}
k_{2}=l_{1}\left(\frac{q}{n^{2}}-\frac{p^{2}}{n^{2}}\right), \\
l_{2}=k_{1}+2 \frac{p}{n} l_{1}
\end{array} \quad \text { and } \quad\left|k_{1} l_{2}-k_{2} l_{1}\right|=1 .\right.
$$

Consider the so-called Pell's equation of the following form:

$$
n^{2} q=\frac{x^{2}-1}{y^{2}}
$$

It was shown by Lagrange that (22) has infinitely many integer solutions (recall that $q$ is not a square of a natural number). Let $x, y$, where $x, y>0$, denote the arbitrary natural solution of this equation. Put

$$
l_{1}=n^{2} y, \quad k_{1}=x-p n y, \quad k_{2}=y\left(q-p^{2}\right), \quad \text { and } \quad l_{2}=x+p n y .
$$

It is a direct consequence of (22) that $x \pm n y \sqrt{q}>0$, hence $k_{1}+\alpha l_{1}>0$. An easy computation shows that each condition in (21) is satisfied by such chosen integers $k_{i}, l_{i}, i=1,2$.

Moreover,

$$
\operatorname{Tr}\left(\begin{array}{ll}
k_{1} & k_{2} \\
l_{1} & l_{2}
\end{array}\right)>2
$$

so it follows from Theorem 1 in [23] that $D \notin \mathfrak{S}$.

(b) It follows from Theorem 14 that any automorphism of $D_{\alpha}$ is elementary algebraic. The automorphisms must also preserve the axis $\{0\} \times \mathbb{C}_{*}$ (when $\alpha>0$, the axis $\mathbb{C} \times\{0\}$ is also preserved). From this piece of information one may conclude that any automorphism of domain $D_{\alpha}$ is of the form

$$
\left(z_{1}, z_{2}\right) \rightarrow\left(a z_{1}, b z_{2}\right), \quad\left(z_{1}, z_{2}\right) \in D_{\alpha},
$$

where $a, b \in \mathbb{C},|a||b|^{\alpha}=1$. Therefore, the functions $u_{+}, u_{-}$given by the formulas

$$
u_{+}\left(z_{1}, z_{2}\right)=\max \left\{\frac{1}{1-\left|z_{1}\right|\left|z_{2}\right|^{\alpha}}, \log \left|z_{1}\right|, \log \left|z_{2}\right|\right\}, \quad \text { when } \alpha>0,
$$

and $u_{-}\left(z_{1}, z_{2}\right)=\max \left\{u_{+}\left(z_{1}, z_{2}\right),-\log \left|z_{2}\right|\right\}$, when $\alpha<0$, satisfy the criterion of Stehlé.

(c) Similarly as before, we easily find from Theorem 14 that the group of automorphisms of $D_{\alpha, r}$ consists of the mappings of the form

$$
\left(z_{1}, z_{2}\right) \rightarrow\left(a z_{1}^{\epsilon}, b z_{2}^{\epsilon}\right), \quad\left(z_{1}, z_{2}\right) \in D_{\alpha, r},
$$

where $\epsilon= \pm 1$ and $a, b \in \mathbb{C},|a||b|^{\alpha}=1$. Thus, the function

$$
u\left(z_{1}, z_{2}\right)=\max \left\{\frac{\left|z_{1}\right|\left|z_{2}\right|^{\alpha}}{r\left|z_{1}\right|\left|z_{2}\right|^{\alpha}-1}, \frac{1}{r-\left|z_{1}\right|\left|z_{2}\right|^{\alpha}}, \log ^{2}\left|z_{1}\right|, \log ^{2}\left|z_{2}\right|\right\},
$$

$\left(z_{1}, z_{2}\right) \in D_{\alpha}$, satisfies the criterion of Stehlé. 


\section{The Case when $D \cap \mathbb{C}_{*}^{2}$ is Hyperbolic}

For a pseudoconvex Reinhardt domain $D$ in $\mathbb{C}^{n}$ let $I(D)$ denote the set of $i=$ $1, \ldots, n$, for which the intersection $\left(\mathbb{C}^{i-1} \times\{0\} \times \mathbb{C}^{n-i}\right) \cap D$ is not hyperbolic (viewed as a domain in $\mathbb{C}^{n-1}$ ). Put

$$
D^{h y p}=D \backslash\left(\bigcup_{i \in I(D)}\left(\mathbb{C}^{i-1} \times\{0\} \times \mathbb{C}^{n-i}\right)\right) .
$$

With a Reinhardt domain $D$ we associate the following constant: $t(D):=$ the number of $i=1, \ldots, n$, such that $D \cap\left(\mathbb{C}^{i-1} \times\{0\} \times \mathbb{C}^{n-i}\right) \neq \emptyset$.

Example 16 Put $D_{1}:=\{(z, w) \in \mathbb{C} \times \mathbb{C}:|z|<1,|z w|<1\}$ and $D_{1}:=\{(z, w) \in$ $\left.\mathbb{C}_{*} \times \mathbb{C}:|z|<1,|z w|<1\right\}$. Then both $D_{1}$ and $D_{2}$ are non-hyperbolic. Observe that $t\left(D_{1}^{\text {hyp }}\right)=1$ and $t\left(D_{2}^{\text {hyp }}\right)=0$.

For a non-hyperbolic pseudoconvex Reinhardt domain $D \subset \mathbb{C}^{2}$ such that $D^{\text {hyp }}$ is hyperbolic, the number $t\left(D^{h y p}\right)$ is equal either to 1 or to 0 .

First we shall focus our attention on the case $t\left(D^{h y p}\right)=1$. We start with the following:

Lemma 17 Let $D$ be a non-hyperbolic, pseudoconvex Reinhardt domain in $\mathbb{C}^{2}$. Assume that the logarithmic image of $D$ contains no affine line and the group of automorphisms of $D^{\text {hyp }}$ is compact. Then $\operatorname{Aut}(D)$ is also compact.

Proof Take any sequence $\left(\varphi_{n}\right)_{n} \subset \operatorname{Aut}(D)$. Since for any $\varphi \in \operatorname{Aut}(D)$ the restriction $\left.\varphi\right|_{D^{h y p}}$ is an automorphism of $D^{\text {hyp }}$ (see, e.g., [11], Theorem 8), we may assume that $\left(\left.\varphi_{n}\right|_{D^{h y p}}\right)_{n}$ is convergent locally uniformly on $D^{\text {hyp }}$. Applying Cauchy's formula we infer that $\left(\varphi_{n}\right)_{n}$ is convergent to some holomorphic function on $D$. Repeating the above argument for the sequence $\left(\varphi_{n}^{-1}\right)_{n}$ immediately gives the desired result.

The problem of a characterization of automorphism groups of bounded Reinhardt domains was studied in [18] (see also [7, 8], and papers referenced there).

The results obtained in [18] and [12], together with remarks from [17], lead us to the description of pseudoconvex hyperbolic Reinhardt domains with $t=1$ and non-compact automorphism group. For our future use we recall here the version formulated in [17].

Theorem 18 (See [12, 18], and [17], Theorem 4) Let D be a hyperbolic, pseudoconvex Reinhardt domain with $t(D)=1$. Then $\operatorname{Aut}(D)$ is non-compact if and only if $D$ is algebraically equivalent to one of the following domains:

(a) $\mathbb{D} \times \mathbb{A}(r, 1)$, where $0 \leq r<1$. In this case, the group of automorphisms consists of the mappings of the form $D \ni\left(z_{1}, z_{2}\right) \rightarrow\left(a\left(z_{1}\right), b\left(z_{2}\right)\right) \in D$, where $a \in \operatorname{Aut}(\mathbb{D})$ and $b \in \operatorname{Aut}(\mathbb{A}(r, 1))$. 
(b) $\left\{\left(z_{1}, z_{2}\right) \in \mathbb{C}^{2}:\left|z_{1}\right|<1,0<\left|z_{2}\right|<\left(1-\left|z_{1}\right|^{2}\right)^{p / 2}\right\}$, $p>0$. In this case, the group of automorphisms consists of the mappings of the form $D \ni\left(z_{1}, z_{2}\right) \rightarrow$ $\left(\alpha \frac{z_{1}-\beta}{1-\bar{\beta} z_{1}}, \gamma \frac{\left(1-|\beta|^{2}\right)^{\frac{p}{2}}}{\left(1-\bar{\beta} z_{1}\right)^{p}} z_{2}\right) \in D$, where $|\alpha|=|\gamma|=1, \beta \in \mathbb{C}$.

(c) $\left\{\left(z_{1}, z_{2}\right) \in \mathbb{C}^{2}: 0<\left|z_{2}\right|<\exp \left(-\left|z_{1}\right|^{2}\right)\right\}$. In this case the group of automorphisms consists of the mappings of the form $D \ni\left(z_{1}, z_{2}\right) \rightarrow\left(\alpha z_{1}+\beta, \gamma \exp \left(-2 \alpha \bar{\beta} z_{1}-\right.\right.$ $\left.\left.|\beta|^{2}\right) z_{2}\right) \in D$, where $|\alpha|=|\gamma|=1, \beta \in \mathbb{C}$.

The following lemma will be also needed:

Lemma 19 (See [11], Theorem 2) Let D, G be pseudoconvex Reinhardt domains in $\mathbb{C}^{2}$. Let $\varphi: D \rightarrow G$ be a proper holomorphic mapping. Assume that the logarithmic images of domains $D$ and $G$ contain no affine lines. Then

$$
\varphi\left(D^{h y p}\right) \subset G^{h y p}
$$

and the restriction

$$
\left.\varphi\right|_{D^{h y p}}: D^{h y p} \rightarrow G^{h y p}
$$

is proper.

Theorem 20 Let $D \subset \mathbb{C}^{2}$ be a pseudoconvex non-hyperbolic Reinhardt domain with $t\left(D^{\text {hyp }}\right)=1$. Assume additionally that the logarithmic image of the domain $D^{\text {hyp }}$ contains no affine lines. Then the group of automorphisms of domain D is compact. In particular, the answer to the Serre problem for the domain $D$ is positive.

Proof In view of Lemma 17 it suffices to prove the theorem for domain $D$ under the additional assumption that $\operatorname{Aut}\left(D^{\text {hyp }}\right)$ is non-compact. Then it is a consequence of Theorem 18 that up to a multiplying and a permutation of components $D$ must have the form

(a) $\left\{\left(z_{1}, z_{2}\right) \in \mathbb{C}^{2}:\left|z_{1} z_{2}^{k}\right|<1,\left|z_{2}\right|<1\right\}$, where $k \in \mathbb{Z}_{>0}$,

(b) $\left\{\left(z_{1}, z_{2}\right) \in \mathbb{C}^{2}:\left|z_{1} z_{2}^{k}\right|<1,\left|z_{2}\right|<\left(1-\left|z_{1} z_{2}^{k}\right|^{2}\right)^{p / 2}\right\}$, for some $p>0, k \in \mathbb{Z}_{>0}$,

(c) $\left.\left\{\left(z_{1}, z_{2}\right) \in \mathbb{C}^{2}:\left|z_{2}\right|<\exp \left(-\left|z_{1} z_{2}^{k}\right|^{2}\right)\right)\right\}, k \in \mathbb{Z}_{>0}$.

Moreover (see Lemma 19), any automorphism of the domain of one of the forms presented above preserves the axis $\mathbb{C} \times\{0\}$. This fact and Theorem 18 lead to the statement that the group of automorphisms of the domain D in all cases (a), (b), and (c) consists of the mappings of the form

$$
D \ni\left(z_{1}, z_{2}\right) \rightarrow\left(a z_{1}, b z_{2}\right) \in D
$$

where $|a|=|b|=1$. Therefore, $\operatorname{Aut}(D)$ is compact in all cases, which is a contradiction.

Let us pass to the remaining case $t\left(D^{\text {hyp }}\right)=0$. An important role in our approach is played by the following result: 
Theorem $21($ See $[12,18]) \operatorname{Aut}(D)=\operatorname{Aut}_{\text {alg }}(D)$ for any pseudoconvex hyperbolic Reinhardt domain $D \subset \mathbb{C}_{*}^{n}$.

Theorem 22 Let $D \subset \mathbb{C}^{2}$ be a non-hyperbolic pseudoconvex Reinhardt domain such that $D^{\text {hyp }}$ is hyperbolic and $t\left(D^{\text {hyp }}\right)=0$. Then $D \in \mathfrak{S}$.

Moreover, the group of automorphisms of $D$ is non-compact if and only if, up to a permutation on components, $D$ is contained in $\mathbb{C} \times \mathbb{C}_{*},\{0\} \times \mathbb{C}_{*} \subset D$ and

$$
\log D=\{(t, s): t<\psi(s), s \in \mathbb{R}\},
$$

where $\psi: \mathbb{R} \rightarrow \mathbb{R}$ is a concave function satisfying the property $\psi(\beta+s)-\psi(s)=$ $\alpha+k s, s \in \mathbb{R}$ for some $\alpha, \beta \in \mathbb{R}, \beta \neq 0, k \in \mathbb{Z}_{*}$.

Proof Using the inclusion $\operatorname{Aut}(D)_{\mid D^{h y p}} \subset \operatorname{Aut}\left(D^{\text {hyp }}\right)$ (Lemma 19) and Theorem 21, we state that any automorphism of $D$ must be algebraic. Moreover, at least one of the axes $\{0\} \times \mathbb{C}_{*}, \mathbb{C}_{*} \times\{0\}$ must be contained in $D$ (otherwise, $D$ would be hyperbolic).

Suppose that both axes are contained in $D$. Then, since any automorphism of $D$ maps the axes onto the axes (use Lemma 19), we see that the group $\operatorname{Aut}(D)$ consists of the mappings of the form

$$
\begin{aligned}
& D \ni\left(z_{1}, z_{2}\right) \rightarrow\left(a z_{1}, b z_{2}\right) \in D \quad \text { or } \\
& D \ni\left(z_{1}, z_{2}\right) \rightarrow\left(a z_{2}, b z_{1}\right) \in D .
\end{aligned}
$$

for suitable $a, b \in \mathbb{C}_{*}$. We shall show that $|a|=|b|=1$ in the case (28). Moreover, we shall prove that there is $R>0$ such that for any automorphism satisfying (29), $|a|=R$ and $|b|=1 / R$. This in particular means that $\operatorname{Aut}(D)$ is compact.

Let us take any $\varphi \in \operatorname{Aut}(D)$ of the form (28).

Assume the contrary, i.e., $(\log |a|, \log |b|) \neq(0,0)$. For $n \in \mathbb{N}$ put $\varphi^{(n)}=\varphi \circ \cdots \circ$ $\varphi \in \operatorname{Aut}(D)$ and $\varphi^{(-n)}=\left(\varphi^{-1}\right)^{(n)}$. Since $\varphi^{(n)}\left(z_{1}, z_{2}\right)=\left(a^{n} z_{1}, b^{n} z_{2}\right) \in D$ for any $\left(z_{1}, z_{2}\right) \in D$, passing to the logarithmic image of the domain $D$ easily shows that $\mathbb{R}(\log |a|, \log |b|)+\log D \subset \log D$. This contradicts the hyperbolicity of $D^{\text {hyp }}$.

Assume that $\varphi:\left(z_{1}, z_{2}\right) \rightarrow\left(a z_{2}, b z_{1}\right)$ is an automorphism of the domain $D$. Repeating the previous reasoning applied to an automorphism $\varphi^{(2)}\left(z_{1}, z_{2}\right)=\left(a b z_{1}\right.$, $a b z_{2}$ ), we immediately see that $|a b|=1$. Therefore, it suffices to show that for any other automorphism $\tilde{\varphi}:\left(z_{1}, z_{2}\right) \rightarrow\left(a_{1} z_{2}, b_{1} z_{1}\right)$ of $D$ the following relations hold: $\left|a_{1}\right|=|a|$ and $\left|b_{1}\right|=|b|$. However, these relations also follow from the above reasoning applied to $\tilde{\varphi} \circ \varphi\left(z_{1}, z_{2}\right)=\left(a_{1} b z_{1}, a b_{1} z_{2}\right)$.

This finishes the proof of the compactness of $\operatorname{Aut}(D)$ in this case.

Suppose that only one axis is contained in $D$, e.g., $\{0\} \times \mathbb{C}_{*} \subset D$. We use the idea applied by P. Pflug and W. Zwonek in [17].

Assume that the group $\operatorname{Aut}(D)$ is non-compact. Note that $D \cap(\mathbb{C} \times\{0\})=\emptyset$. As before, using the fact that automorphisms preserve the axis (Lemma 19), we see that $\operatorname{Aut}(D)$ consists of the mappings of the form

$$
\Phi=\Phi_{a, b, k, \epsilon}:\left(z_{1}, z_{2}\right) \rightarrow\left(a z_{1} z_{2}^{k}, b z_{2}^{\epsilon}\right)
$$

for some $a, b \in \mathbb{C}_{*}, \epsilon= \pm 1$ and $k \in \mathbb{Z}$. 
$(\dagger)$ We shall show that there exists an automorphism $\Phi_{a, b, k, \epsilon}$ of the domain $D$ with $|b| \neq 1$ and $\epsilon=1$ (then also $k \neq 0$ ).

First observe that there is an automorphism of the domain $D$ of the form (30) with $k \neq 0$. To show this, repeat the argument from the first part of the proof to observe that $|a|=|b|=1$ for any automorphism $\Phi_{a, b, 0,1}$. Moreover, there exists $R>0$ such that $|a|=1,|b|=R$ for any automorphism $\Phi_{a, b, 0,-1}$. So if there were no automorphisms of the form (30) with $k \neq 0$, then the group $\operatorname{Aut}(D)$ would be compact.

If there is an automorphism $\Phi$ of $D$ of the form (30) with $\epsilon=1$ and $k \neq 0$, we are done. Actually, $\Phi^{(n)}\left(z_{1}, z_{2}\right)=\left(a^{n} b^{k n(n-1) / 2} z_{1} z_{2}^{n k}, b^{n} z_{2}\right)$, and using the hyperbolicity of $D^{\text {hyp }}$ once again, we find that $|b| \neq 1$.

Thus, to prove $(\dagger)$ it suffices to consider the case when any automorphism of the form (30) with $k \neq 0$ satisfies $\epsilon=-1$. Since $\Phi_{a, b, k,-1}^{(2 n)}\left(z_{1}, z_{2}\right)=\left(\left(a^{2} b^{k}\right)^{n} z_{1}, z_{2}\right)$, $n \in \mathbb{Z}$, we see that $\left|a^{2} b^{k}\right|=1$. So there is an automorphism $\Phi_{\tilde{a}, \tilde{b}, \tilde{k},-1}$ such that $|\tilde{b}| \neq|b|$ or $\tilde{k} \neq k$ (as $\operatorname{Aut}(D)$ is non-compact). Let us compute

$$
\Phi_{a, b, k,-1} \circ \Phi_{\tilde{a}, \tilde{b}, \tilde{k},-1}=\Phi_{a \tilde{a} \tilde{b}^{k}, \tilde{b}, \tilde{k}-k, 1} .
$$

If $|\tilde{b}| \neq|b|$, then the statement is clear. Otherwise, $k \neq \tilde{k}$. But this contradicts the assumption that any automorphism of the form (30) with $k \neq 0$ satisfies $\epsilon=-1$.

So we have shown $(\dagger)$.

The properties of pseudoconvex Reinhardt domains imply that for any $s \in \mathbb{R}$ there is (exactly one) $\psi(s) \in \mathbb{R}$ such that $(\psi(s), s) \in \partial \log D$. Moreover, it is an immediate consequence of the inclusion $\{0\} \times \mathbb{C}_{*} \subset D$ and the convexity of $\log D$ that the function $\psi(\cdot)$ is concave. Put

$$
v\left(z_{1}, z_{2}\right)=\log \left|z_{1}\right|-\psi\left(\log \left|z_{2}\right|\right) .
$$

For fixed $\left(z_{1}, z_{2}\right) \in D \cap \mathbb{C}_{*}^{2}$ put $(t, s)=\left(\log \left|z_{1}\right|, \log \left|z_{2}\right|\right)$. Let $\Phi=\Phi_{a^{\prime}, b^{\prime}, k^{\prime}, \epsilon}$ be an arbitrary automorphism given by the formula (30).

Denote $\left(t^{\prime}, s^{\prime}\right)=\left(\log \left|\Phi_{1}(z)\right|, \log \left|\Phi_{2}(z)\right|\right)$. It is seen that $\left(t^{\prime}, s^{\prime}\right)=\left(\log \left|a^{\prime}\right|+\right.$ $\left.t+k^{\prime} s, \log \left|b^{\prime}\right|+\epsilon s\right)$.

Since $\left.\Phi\left((\partial D) \cap \mathbb{C}_{*}^{2}\right)\right)=(\partial \Phi(D)) \cap \mathbb{C}_{*}^{2}$, we see that

$$
\left(\psi\left(s^{\prime}\right), s^{\prime}\right)=\left(\log \left|a^{\prime}\right|+\psi(s)+k^{\prime} s, \log \left|b^{\prime}\right|+\epsilon s\right) .
$$

Therefore, $\psi\left(s^{\prime}\right)-t^{\prime}=\psi(s)-t$. In other words,

$$
v \circ \Phi=v \quad \text { for any } \Phi \in \operatorname{Aut}(D) .
$$

Define

$$
u\left(z_{1}, z_{2}\right)=\max \left\{\log \left|z_{2}\right|,-\log \left|z_{2}\right|,-\left(v\left(z_{1}, z_{2}\right)\right)^{-1}\right\}, \quad\left(z_{1}, z_{2}\right) \in D .
$$

A direct calculation (approximate and compute the Levi form) shows that $u$ is plurisubharmonic. Moreover, $u$ is an exhaustion function for $D$. In view of (32) $u$ satisfies the criterion of Stehlé. 
Using $(\dagger)$ and the property (31), once again we find that $\psi(\log |b|+s)-\psi(s)=$ $\log |a|+k s$. From this we obtain the desired properties of the function $\psi$.

Conversely, having a domain $D \subset \mathbb{C} \times \mathbb{C}_{*}$ satisfying (27) one may easily see that the mapping $\Phi$ given by the formula (30) with $b=e^{\beta}, a=e^{\alpha}, \epsilon=1$ is an automorphism of the domain $D$. The investigation of $\Phi^{(n)}$ immediately proves the non-compactness of $\operatorname{Aut}(D)$.

\section{$6 \mathbb{C} \times \mathbb{C}_{*}$}

In 1977 a negative answer to the Serre problem was given by H. Skoda, who proved that $\mathbb{C}^{2} \notin \mathfrak{S}$. This construction was improved in [2] by J.P. Demailly, who proved that polynomial automorphisms of $\mathbb{C}^{2}$ may serve as the transition function. Later, in [3], J.P. Demailly constructed a counterexample to the Serre problem with a plane or a disc as a base.

Let us recall here this construction. The base $\Omega$ is a domain containing $3 \mathbb{D}$. Put $\Omega_{0}=\Omega \backslash\{-1,1\}, \Omega_{1}=\Omega_{0} \cup\{1\}, \Omega_{2}=\Omega_{0} \cup\{-1\}$. The transition functions $\tau_{i, j}$ : $\Omega_{i} \times \mathbb{C}^{2} \rightarrow \Omega_{j} \times \mathbb{C}^{2}, i \neq j$ of the fiber bundle $E$ are defined as follows:

$$
\begin{aligned}
& \tau_{0,1}\left(x ; z_{1}, z_{2}\right)=\left(x ; z_{1}, z_{2} \exp \left(z_{1} u(x)\right),\right. \\
& \tau_{0,2}\left(x ; z_{1}, z_{2}\right)=\left(x, z_{1} \exp \left(z_{2} u(x)\right), z_{2}\right)
\end{aligned}
$$

and $\tau_{1,2}=\tau_{0,1}^{-1} \circ \tau_{0,2}$, where $u(x)=\exp \left(\frac{1}{x^{2}-1}\right)$. It is clear that any plurisubharmonic function $V$ on $E$ induces plurisubharmonic $V_{j}$ such that $V_{j}=V_{k} \circ \tau_{k, j}$. The idea of the proof relied upon comparing the maximum of the functions $V_{j}$ over the polydiscs $\frac{1}{2} \mathbb{D} \times(r \mathbb{D})^{2}, r \gg 1$. More precisely, it was shown that

$$
M\left(V_{0}, \frac{1}{2} \mathbb{D}, \exp (r / 32)\right) \leq M\left(V_{0}, \frac{1}{2} \mathbb{D}, \exp \left(\log ^{3} r\right)\right)+C, \quad r \gg 1,
$$

where $M(V, \omega, r)=\max _{\omega \times(r \mathbb{D})^{2}} V$ and the constant $C$ does not depend on $r$. The key role was played by the logarithmic convexity of the functions

$$
(\rho, r) \rightarrow M(V, \rho \mathbb{D}, r), \quad V \in \mathcal{P} \mathcal{S H}\left(\Omega \times \mathbb{C}^{2}\right) .
$$

Direct computation allows us to obtain the logarithmic convexity of the function $(\rho, r) \rightarrow \max _{\rho \mathbb{D} \times r \mathbb{D} \times \mathbb{A}(r)} \tilde{V}$ for any $\tilde{V} \in \mathcal{P} \mathcal{S H}\left(\Omega \times \mathbb{C} \times \mathbb{C}_{*}\right)$. Therefore, considering $\tilde{M}$ instead of $M$, where $\tilde{M}(V, \omega, r)=\max _{\omega \times r \mathbb{D} \times \mathbb{A}(r)} V$, and repeating the reasoning from Demailly's paper, we may replace $M$ by $\tilde{M}$ in the inequality (33). This, together with the above-mentioned logarithmic convexity of $\tilde{M}$, immediately shows that $\mathbb{C} \times \mathbb{C}_{*}$ does not belong to $\mathfrak{S}$.

\section{Proofs of the Main Theorems}

Proof of Theorem 1 The result follows from Theorems 12, 15, 20, and 22. 
Proof of Theorem 3 It is clear that if $\log D$ contains an affine line, then the group $\operatorname{Aut}(D)$ is non-compact.

In the case when $\log D$ contains no affine lines (i.e., $D^{\text {hyp }}$ hyperbolic) the result follows immediately from Theorems 20 and 22.

Acknowledgements The author expresses his thanks to the referee for his valuable suggestions to improve the shape of the original paper. Also, I would like to express my gratitude to professor Włodzimierz Zwonek for helpful discussions.

\section{References}

1. Coeuré, G., Loeb, J.-J.: A counterexample to the Serre problem with a bounded domain in $\mathbb{C}^{2}$ as fiber. Ann. Math. 122, 329-334 (1985)

2. Demailly, J.P.: Différents exemples de fibré holomorphes non de Stein. In: Séminaire P. Lelong-H. Skoda. Lecture Notes in Math., vol. 694, pp. 15-41. Springer, Berlin (1976-1977)

3. Demailly, J.P.: Un exemple de fibré holomorphe non de Stein á fibre $C^{2}$ au-dessus du disque ou du plan. In: Séminaire P. Lelong, P. Dolbeault, H. Skoda (Analyse). Lecture Notes in Math., vol. 1198, pp. 88-97. Springer, Berlin (1983/84)

4. Edigarian, A., Zwonek, W.: Proper holomorphic mappings in some class of unbounded domains. Kodai Math. J. 22, 305-312 (1999)

5. Grauert, H.: Charakterisierung der holomorph-vollständigen komplexen Räume. Math. Ann. 129, 233-259 (1955)

6. Hörmander, L.: An Introduction to Complex Analysis in Several Variables. 3rd edn. North-Holland Mathematical Library, vol. 7. North-Holland, Amsterdam (1990)

7. Isaev, A.V., Krantz, S.G.: Domains with non-compact automorphism group: a survey. Adv. Math. 146, 1-38 (1999)

8. Isaev, A.V., Kruzhilin, N.G.: Proper holomorphic maps between Reinhardt domains in $\mathbb{C}^{2}$. Mich. Math. 54, 33-64 (2006)

9. Jarnicki, M., Pflug, P.: First Steps in Several Complex Variables: Reinhardt Domains, EMS Textbooks in Mathematics. European Mathematical Society (EMS), Zürich (2008)

10. Kosiński, Ł.: Proper holomorphic mappings in the special class of Reinhardt domains. Ann. Pol. Math. 92, 285-297 (2007)

11. Kosiński, Ł.: Proper holomorphic mappings between Reinhardt domains in $\mathbb{C}^{2}$. Mich. Math. J. 58(3), 711-721 (2009)

12. Kruzhilin, N.G.: Holomorphic equivalence of hyperbolic Reinhardt domains. Math. USSR Izv. 32, 15-38 (1989)

13. Mok, N.: Le problème de Serre pour les surfaces de Riemann. C. R. Acad. Sci. Paris, Sér. A-B 290(4), 179-180 (1980)

14. Mok, N.: The Serre problem on Riemann surfaces. Math. Ann. 258, 145-168 (1981)

15. Narasimhan, R.: A note on Stein spaces and their normalizations. Ann. Sc. Norm. Super. Pisa 16, 327-333 (1962)

16. Oeljeklaus, K., Zaffran, D.: Steinness of bundles with fiber a Reinhardt bounded domain. Bull. Soc. Math. Fr. 134(4), 451-473 (2006)

17. Pflug, P., Zwonek, W.: The Serre problem with Reinhardt fibers. Ann. Inst. Fourier 54, 129-146 (2004)

18. Shimizu, S.: Automorphisms and equivalence of bounded Reinhardt domains not containing the origin. Tohoku Math. J. (40) 1, 119-152 (1988)

19. Shimizu, S.: Holomorphic equivalence problem for a certain class of unbounded Reinhardt domains in $\mathbb{C}^{2}$. II. Kodai Math. J. 15, 430-444 (1992)

20. Skoda, H.: Fibré holomorphes á base fibre et á fibre de Stein. Invent. Math. 43, 97-107 (1977)

21. Soldatkin, P.A.: Holomorphic equivalence of Reinhardt domains in $\mathbb{C}^{2}$. Izv. Ross. Akad. Nauk Ser. Mat. Phys. 66(6), 187-222 (2002) (Russian) translation in Izv. Math. 66(6), 1271-1304 (2002)

22. Stehlé, J.-L.: Fonctions plurisousharmoniques et convexité holomorphe de certaines fibrés analytiques. In: Lelong, P., Doulbeaut, P., Skoda, H. (eds.) Séminaire Pierre Lelong (Analyse), Année 1973/74. Lecture Notes in Mathematics, vol. 474, pp. 155-179 Springer, Berlin (1975)

23. Zaffran, D.: Holomorphic functions on bundles over annuli. Math. Ann. 341(4), 717-733 (2008)

24. Zwonek, W.: On hyperbolicity of pseudoconvex Reinhardt domains. Arch. Math. 72, 304-314 (1999) 